\title{
CONF-950750--33
}

UCRL-JC-119362

PREPRINT

\section{Integrated Power Conditioning for Laser Diode Arrays}

\author{
R. L. Hanks, H. C. Kirbie, M. A. Newton and M. S. Farhoud* \\ Lawrence Livermore National Laboratory, University of California \\ P.O. Box 5508, L-441, Livermore California 94550, USA \\ *Department of Electrical Engineering, University of Texas at Arlington \\ Arlington, Texas 76019, USA
}

This paper was prepared for submittal to the

Proceedings of the 10th IEEE Pulsed Power Conference

Albuquerque, NM

July 10-13, 1995

June 30, 1995

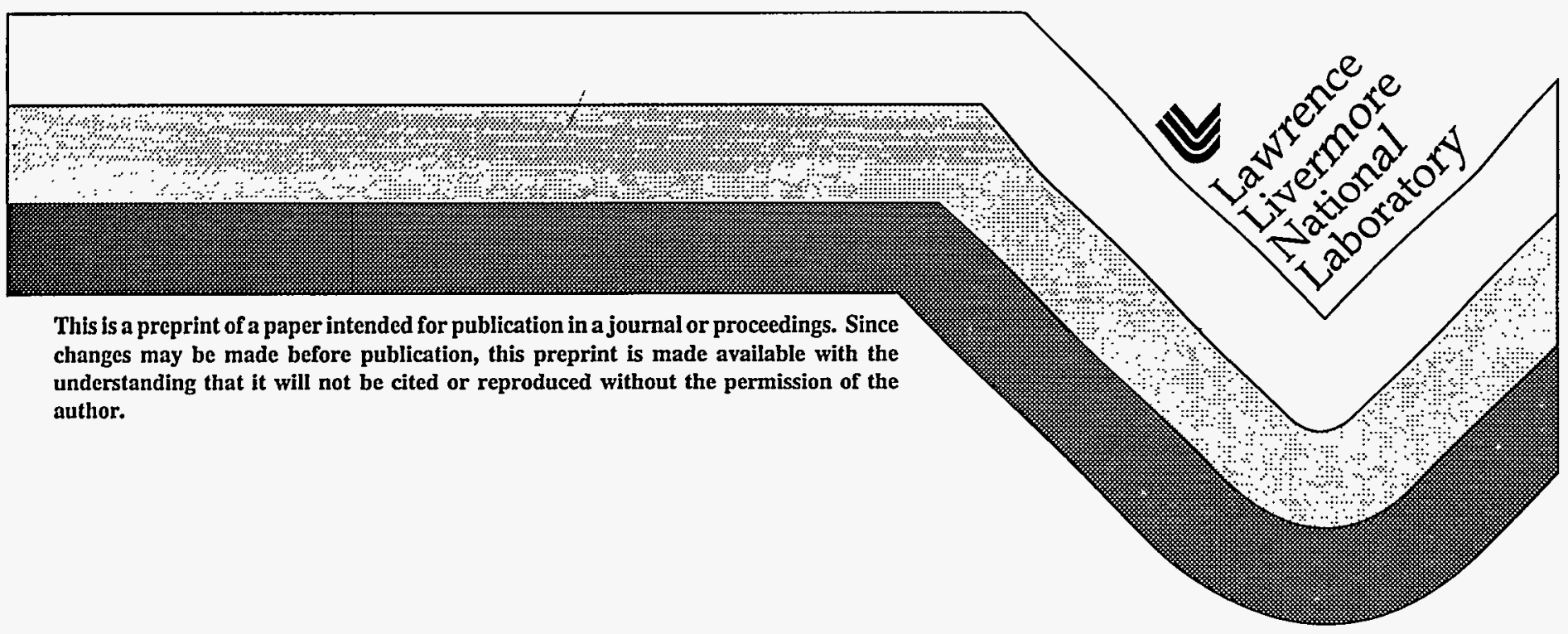




\section{DISCLAIMER}

Portions of this document may be illegible in electronic image products. Images are produced from the best available original document. 


\title{
INTEGRATED POWER CONDITIONING FOR LASER DIODE ARRAYS
}

\author{
R.L. Hanks, H.C. Kirbie, M.A. Newton \\ Lawrence Livermore National Laboratory, Livermore, California 94550 \\ M.S. Farhoud
}

Department of Electrical Engineering, University of Texas at Arlington, Arlington, Texas 76019

\section{Introduction}

For several years, the Laser Program at LLNL has been designing and building a variety of specialized compact laser systems ${ }^{1}$. They offer extremely high energy densities for their size and are primarily used in medical and military applications where a high premium is placed on both reliability and compactness. These systems are based upon a $2 \mathrm{~cm}-\mathrm{x}-2 \mathrm{~cm}$ liquid cooled wafer (Fig. 1) that supports laser diode bars mounted along one edge. Depending upon the application, the laser diodes are fabricated to emit in the 640-nm to $970-\mathrm{nm}$ range while being driven by electrical current pulses of 80-160 A. In some applications, their output is used either to directly irradiate an object or to pump a solid state rod or slab laser. These systems operate at high average power levels and require innovative modular cooling system in which the diode bars are mounted above a micro channel cooling strip ${ }^{2}$.

Wafer size

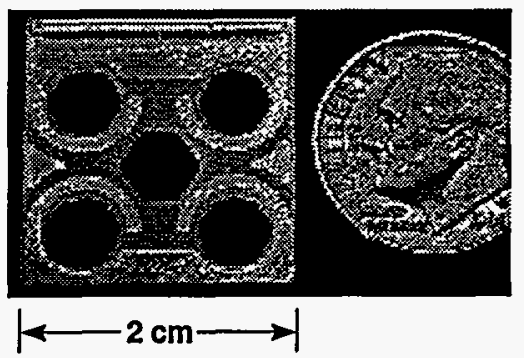

Specifications

$\begin{array}{ll}\text { Wafer thickness } & =1.55 \mathrm{~mm} \\ \text { Number of diodes } & \mathbf{1 1 2 5} \\ \text { Nominal current } & =120-160 \mathrm{~A} \\ \text { Nominal wafer voltage } & =2.2 \mathrm{~V} \bigcirc 120 \mathrm{~A} \\ \text { Maximum duty cycle } & =25 \% \\ \text { Optical wave length } & =800 \mathrm{~nm} \\ \text { Average optical power/wafer } & =23.8 \mathrm{~W} \\ \text { (@ 120 A with } 25 \% \text { duty cycle) } & \\ \text { Electrical-to-optical efficiency } & =36 \% \\ \text { Coolant flow rate } & \cong 2 \mathrm{~cm}^{3} / \mathrm{s}\end{array}$

Figure 1. Wafer photograph (left) comparing the wafer size with a dime. The top edge of the wafer is lined with laser diodes. The table (right) lists selected mechanical, electrical, and optical specifications.

The modulators once used to drive the laser diode arrays have not enjoyed the same level of development as the laser assembly. . As a result the modulators offer power densities several orders of magnitude lower than that of the laser diode array which makes them physically large when compared to the typical laser system. Our goal was to increase the power density and reduce the size of the modulator while at the same time improving regulation and circuit protection. We have demonstrated a new compact power management system that can drive laser diode arrays at peak currents of up to $160 \mathrm{~A}$, at peak voltages of up to $120 \mathrm{~V}$, and maximum duty cycle of $25 \%$. The small size of the power system allows the switching to take place at the laser diode array thus quickly responding to changes in current. As a result variations in lasér output intensity are minimized. Also unique to this system is the concept of keeping to a minimum the number of electrical connections to the outside world to reduce the possibility of ground loop problems and to simplify system integration. The only external connections are prime DC power and a fiber-optic command pulse.

One unlikely but possible fault scenario associated with a laser diode array involves the delamination of a laser bar from its microchannel cooler. If this were to occur an arc could be initiated which in a few seconds could fracture the microchannel substrate. This would result in a loss of coolant and possible damage to the remainder of the laser diode array. To guard against this possible fault condition a crowbar circuit and associated sensing circuitry has also been incorporated into the design.

\section{Approach}

One of our modulator goals was the regulation of laser current to within $1 \%$ for the entire pulse duration. Our approach is the use of a linear power device, operating near saturation, in a constant current feedback configuration. This configuration allows for the use of a small capacitor bank because the feedback system can compensate for both load and supply variations while holding the laser current constant. The desire for linear operation eliminated the possibility of using thyristor type devices.

Based upon our requirements for high efficiency and speed coupled with simplified drive circuitry, MOSFETs and IGBTs were chosen for evaluation. These two devices are both voltage controlled and have almost identical drive requirements. The devices chosen for evaluation included the model IXTH50N20 N-channel enhancement mode power MOSFET. This is a $200 \mathrm{~V} / 50 \mathrm{~A}$ device with a 
maximum average power dissipation of $300 \mathrm{~W}$ and an on resistance of $45 \mathrm{~m} \Omega$. The drain to source rise and fall times are typically 15 ns and 16 ns respectively. Two of these devices were used in parallel to achieve the desired power handling capabilities. The other device chosen was an Advanced Power Technology model APT90GF090JN N-channel enhancement mode high voltage power IGBT. This is a $900 \mathrm{~V} / 90$ A device with a maximum average power rating of $415 \mathrm{~W}$ and an on resistance of $26 \mathrm{~m} \Omega$. The collector to emitter rise and fall times are typically $50 \mathrm{~ns}$ and $400 \mathrm{~ns}$ respectively.

In the case of our requirement for a solid state crowbar device the primary requirements were the ability to handle single event current peaks in excess of $2200 \mathrm{~A}$, and fast turn on capability. Based on these requirements the MCT promised the best balance of conduction drop, junction temperature, $d v / d t$, $\mathrm{di} / \mathrm{dt}$, and surge current rating. MCTs are saturated switches that have the ability to be both turned on and turned off while operating at very high peak and average currents. The selected device was the Harris model MCTV75P60E1 P-Type MCT. This is a 600 V / 75 A device with a non-repetitive peak cathode current rating of $2000 \mathrm{~A}$. The current turn-on delay time is $300 \mathrm{~ns}$ and the current rise time is quoted as $200 \mathrm{~ns}$.

\section{Circuit Description}

Referring to Figure 2 shows a schematic of the modulator and crowbar circuitry in addition to the laser diode array. An optical input into A1 generates an electrical signal that determines when and for how long the laser pulse will occur. This is then fed into a comparator (U4) which inverts and shapes the signal. Potentiometer R13 adjusts the current through the laser diode array during the duration of a laser pulse. U5 provides the constant current regulation in conjunction with current viewing resistor $\mathrm{R} 20$. It also drives the power MOSFETs (Q2, Q3) or IGBT depending on the modulator configuration which in turn drive the laser diode array.

The crowbar circuit begins by differentially measuring (U3) the voltage drop across the laser diode array. A peak detector (U2) increases the circuit's sensitivity to transient voltage spikes which could be the first indication of a possible fault condition. That signal is then fed into a comparator (U1) which determines the fault detection threshold and drives Q1 which in turn triggers the two parallel MCTs (CR6, CR7). If a fault were to occur the MCTs provide a short circuit current path around the laser diode array and modulator thus shunting current away and providing protection.

All the circuitry shown in Figure 2 with the exception of the laser diode array fit on a printed circuit board that measures $6.5^{n}$ by $3.5^{n}$ by $0.6^{\prime \prime}$. This compares favorably with the laser diode array that we used in our testing that measures $4.8^{n}$ by $1.3^{n}$ by $1.0^{n}$.

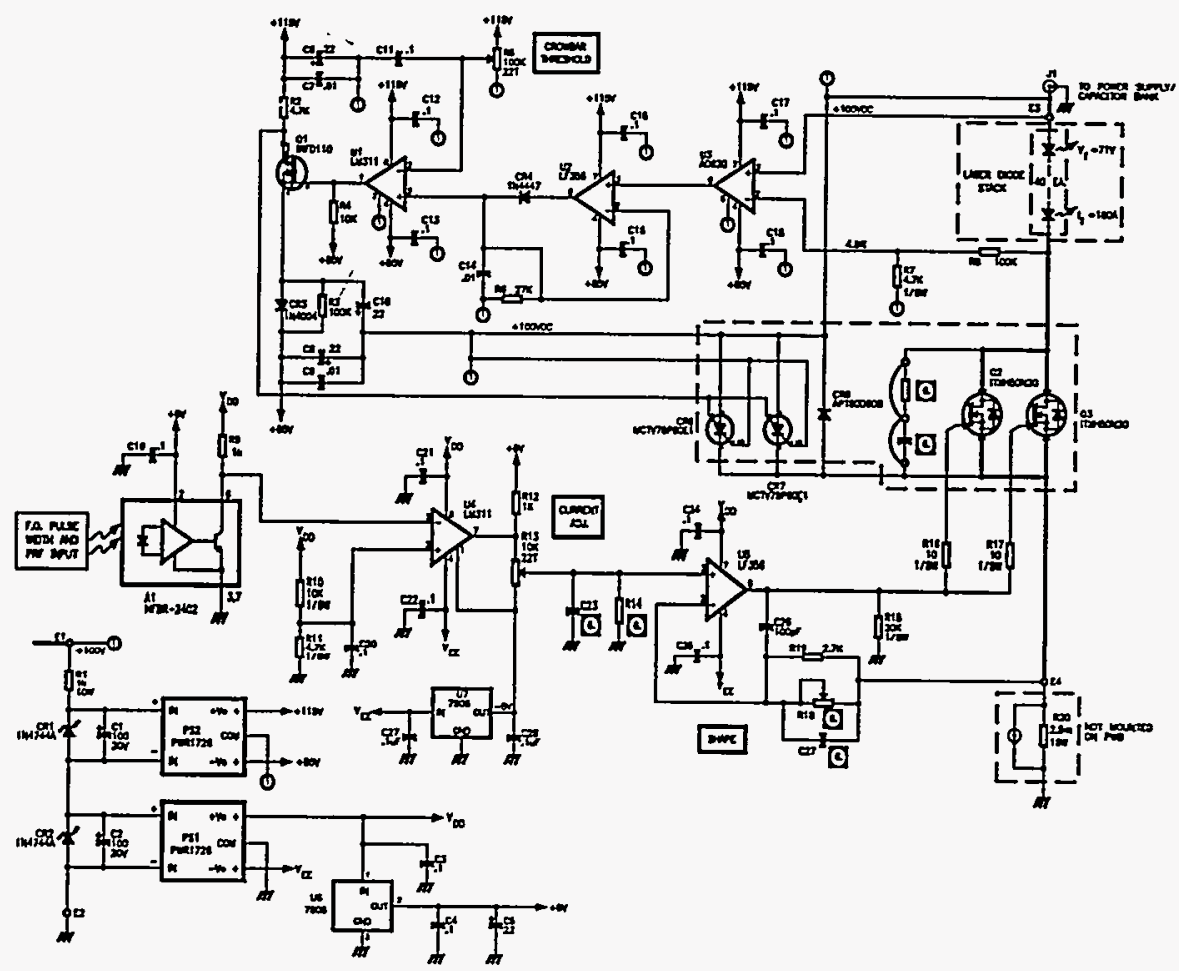

Figure 2. Electrical schematic diagram showing modulator circuit and laser diode array. 
Evaluation

Initial testing of the modulator circuit was conducted using a series stack of silicon power diodes as a dummy load. This was done so as not to risk damaging a valuable laser diode array during initial modulator check out. The number of series diodes in the dummy load was determined such that their forward voltage drop and on resistance produced the same total voltage drop as a typical laser diode array at normal operating current levels. Figure 3 shows three key waveforms associated with the modulator driving a diode load. Figure $3 \mathrm{a}$ and $\mathrm{c}$ show constant voltage and current respectively across the diode load. Figure $3 \mathrm{~b}$ shows the time varying voltage drop across the MOSFETs, which is compensating for supply voltage and/or load impedance variations. Figure 4 shows a comparative plot of the linear operating area (LOA) associated with the modulator configured using MOSFETs and IGBTs. One should note the almost identical performance of the devices. This is a result of similar conduction drops across the devices near saturation.
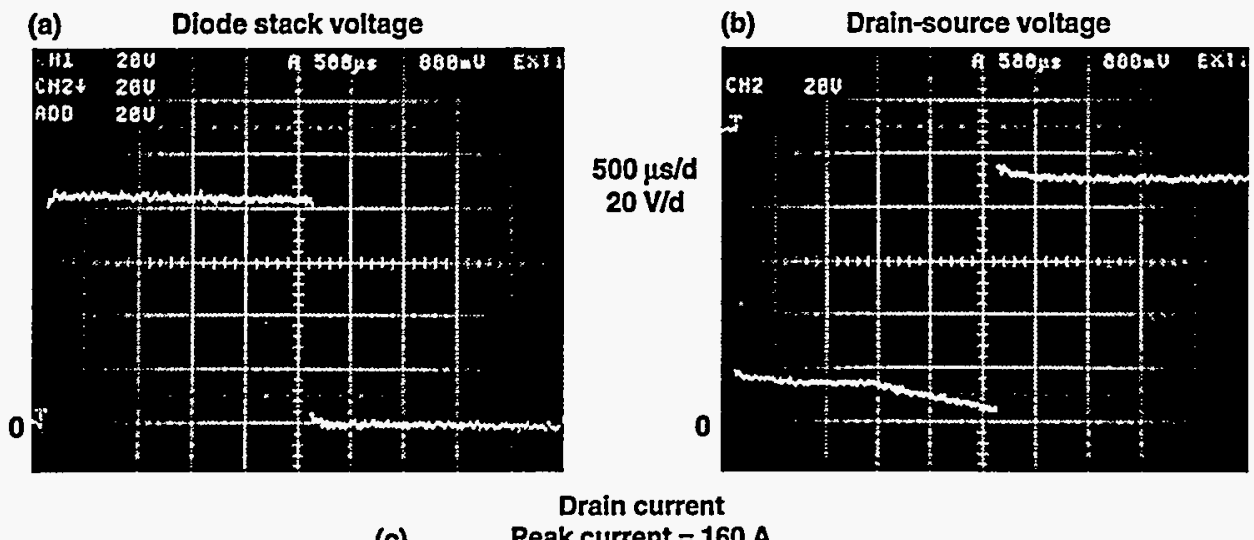

(c) Peak current $=160 \mathrm{~A}$

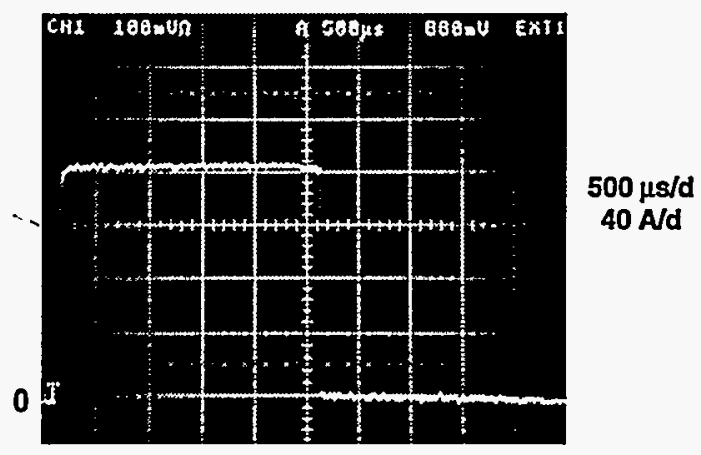

Figure 3. Collection of oscilloscope photographs showing the diode stack voltage (a), the MOSFET drain-source voltage (b), and the diode stack current, which is the same as the drain current (160 A).

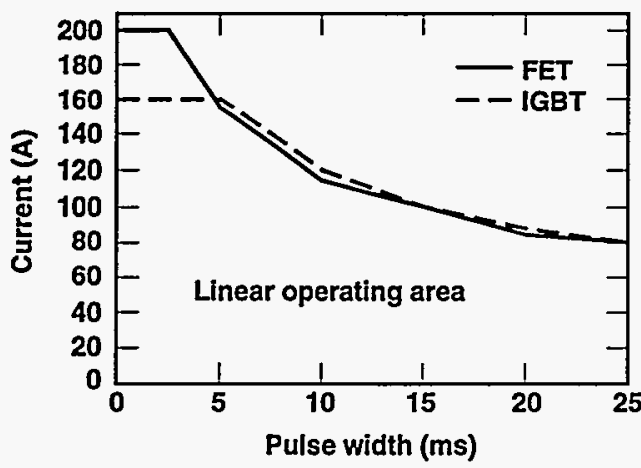

Typical performance summary Source voltage $\quad=100 \mathrm{~V}$ Pulse current $\quad=120-160 \mathrm{~A}$ Pulse width $\quad=100 \mu \mathrm{s}-25 \mathrm{~ms}$ Nominal droop $\quad=1 \%$ Maximum duty cycle $\quad=25 \%$ Maximum PRF $\quad=1.0 \mathrm{kHz}$ Maximum average power $\quad=4.1 \mathrm{~kW}$ Efficiency at maximum power $=-90 \%$

Figure 4. Plot (left) showing a boundary between regulated and unregulated operation for variations in current and pulse width. Operating points below the boundary line will have regulated current pulses. The table (right) is a list of selected performance values. 
To test the sensitivity and response time of the crowbar circuit a common $8 \mathrm{~A}$ fuse was placed in series with the diode load. The current through the load was increased until the fuse began to open. In less than $1 \mu \mathrm{s}$ this fault was detected and two MCTs effected a short circuit across the load and MOSFETs thus isolating them from the capacitor bank, power supply, and any energy stored in connecting cables. The peak current was limited to $2236 \mathrm{~A}$ by a combination of the inductance of the cable connecting the capacitor bank to the modulator and the system capacitance. This abnormally high current that results also causes protection circuitry in the capacitor bank to crowbar and the DC power supply to shut down.

\section{P-Spice Simulations}

The modulator, crowbar and laser diode array circuits were each modeled using P-Spice simulation software. They were then interconnected together thus simulating the interactions of the entire system. These simulations were used to verify the proper operation as well as parasitic and component tolerance sensitivity of the system. Figure 5 compares the P-Spice simulation to actual data for normal operation. The slight difference in rise time characteristics is probably due to stray series inductance. Figure 6 shows the current trace associated with an actual crowbar event compared to the P-Spice simulation. The overall shape of the waveforms show good agreement with a difference in peak current of $14 \%$.
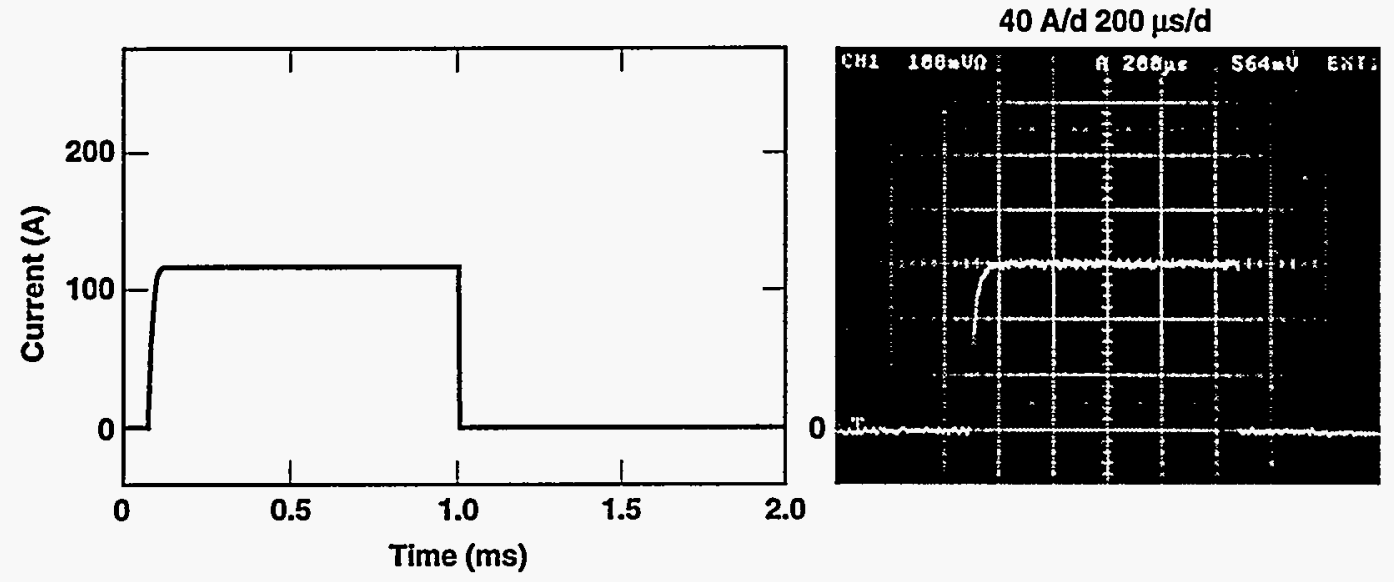

Figure 5. P-Spice simulation (left) ànd measured data (right) show good agreement for a typical $120 \mathrm{~A}$ current pulse.
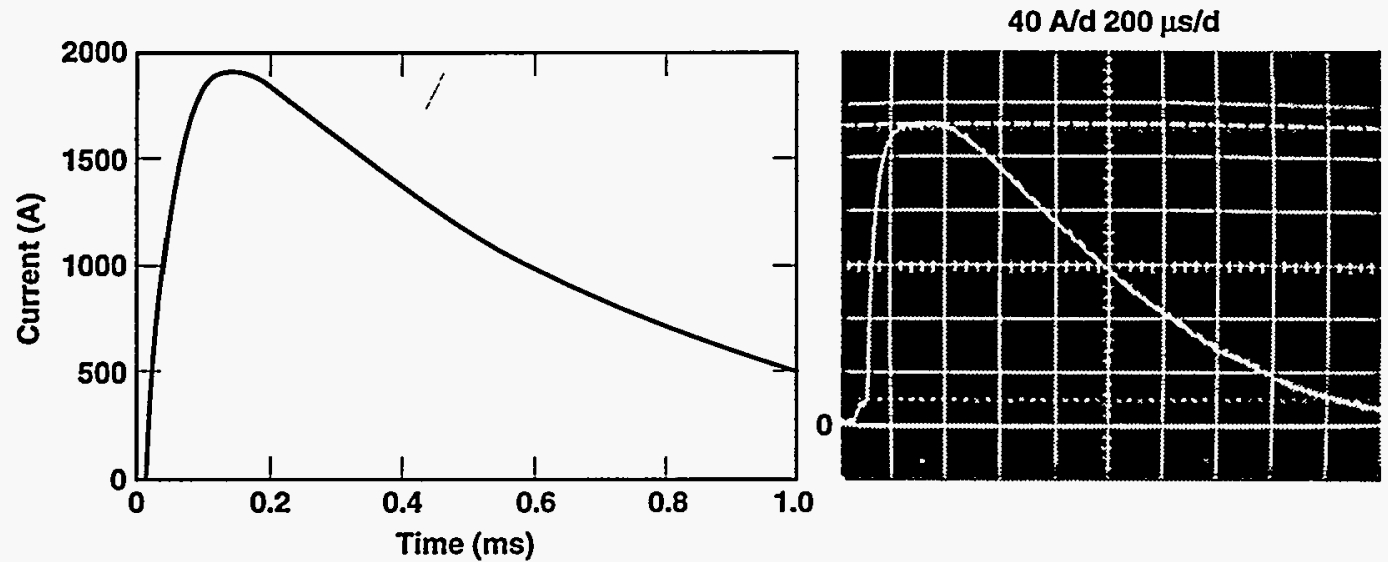

Figure 6. Comparison between P-Spice simulation (left) and measured data (right) for a crowbar action. 
In addition, the current feedback circuitry's ability to cope with abrupt variations in power supply voltage was also tested through modeling. The approach used was to step the supply voltage down from $100 \mathrm{~V}$ to $90 \mathrm{~V}$ for $100 \mu \mathrm{s}$ with transition times of $1.0 \mu \mathrm{s}$. This event was synchronized with the modulator to occur in the center of a $180 \mu \mathrm{s}, 120 \mathrm{~A}$ output pulse. The results show a $8.3 \%$ variation in output current for a duration of $2.0 \mu \mathrm{s}$ occurring at the power supply transitions, with the modulator demonstrating good damping characteristics, as shown in Figure 7.

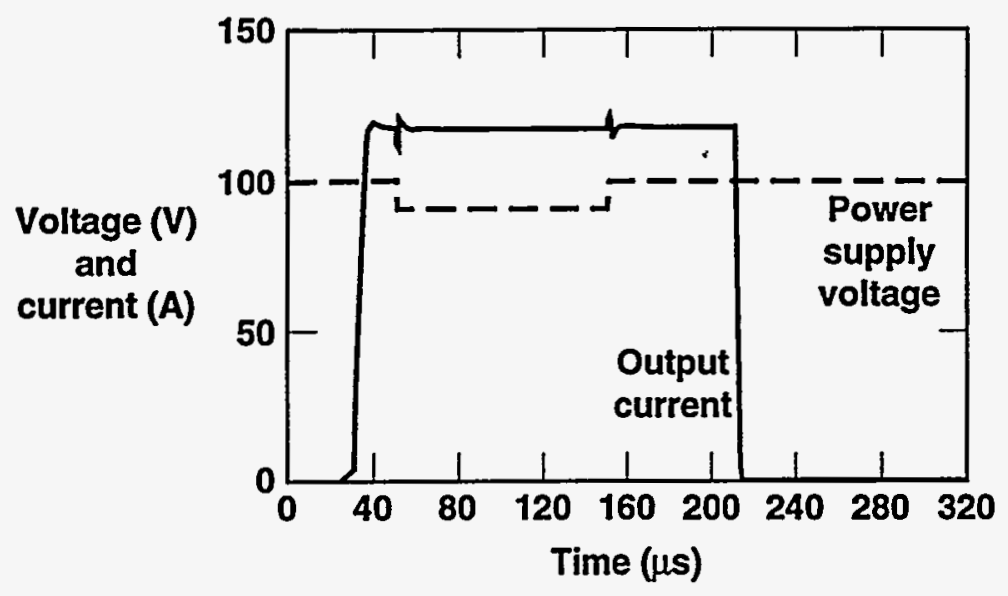

Figure 7. Load current regulation was modeled as function of supply voltage using P-Spice. Here the supply voltage was decreased by $10 \mathrm{~V}$ for $100 \mu$ s with the modulator demonstrating good transient regulation.

\section{Tests with a Laser}

Once we had gained sufficient confidence in the operation and dependability of the modulator, it was time to drive a laser diode array. The array that was available for our testing consisted of 58 wafers; however, each wafer only contains 70 parallel laser diodes instead of the normal 125. As a result the peak current for this laser diode array is only $60 \mathrm{~A}$, at a supply voltage of $125 \mathrm{~V}$. The output of the laser diode array was measured using an optical detector and compared to the drive current waveform, as shown in Figure 8. One should note the good agreement in between the two signals in terms of rise and fall times, pulse width, and temporal response. The'apparent droop of the optical signal in relation to the current is not real and is a result of the detector's poor response to long optical pulses.

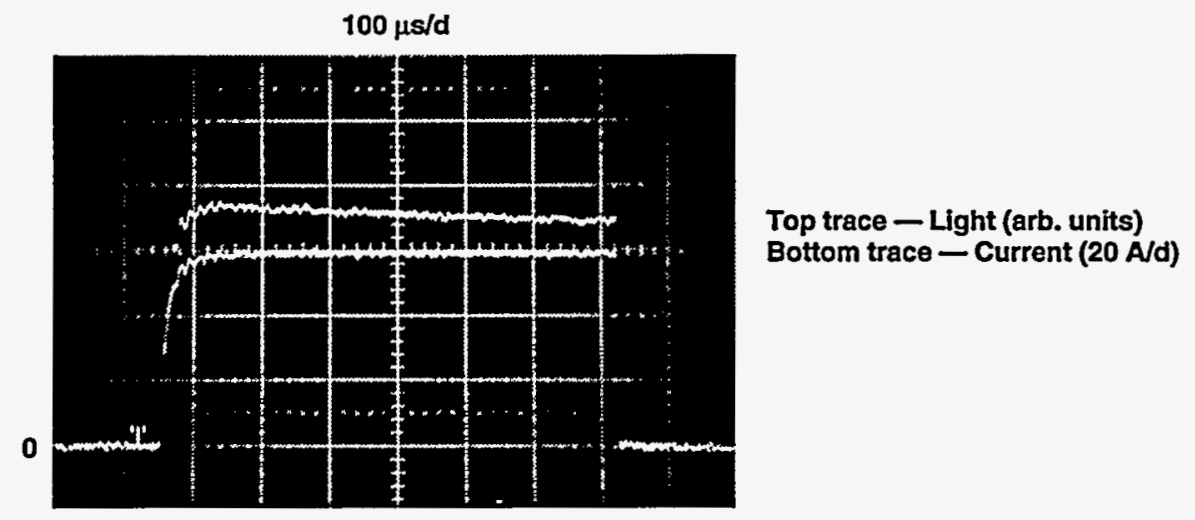

Figure 8. Oscilloscope photograph overlaying the current through a laser diode stack with a measurement of the laser light. 
Figure 9 shows the spectral output of the laser diode array at three different drive levels. Variations in the spectra are the result of laser diode related thermal effects. As the diode laser temperature increases diode emission shifts to longer wavelengths. This is due to expansion of the semiconductor lattice and a decrease in band gap at elevated temperatures. This demonstrates the need for good current regulation to maintain spectral purity.

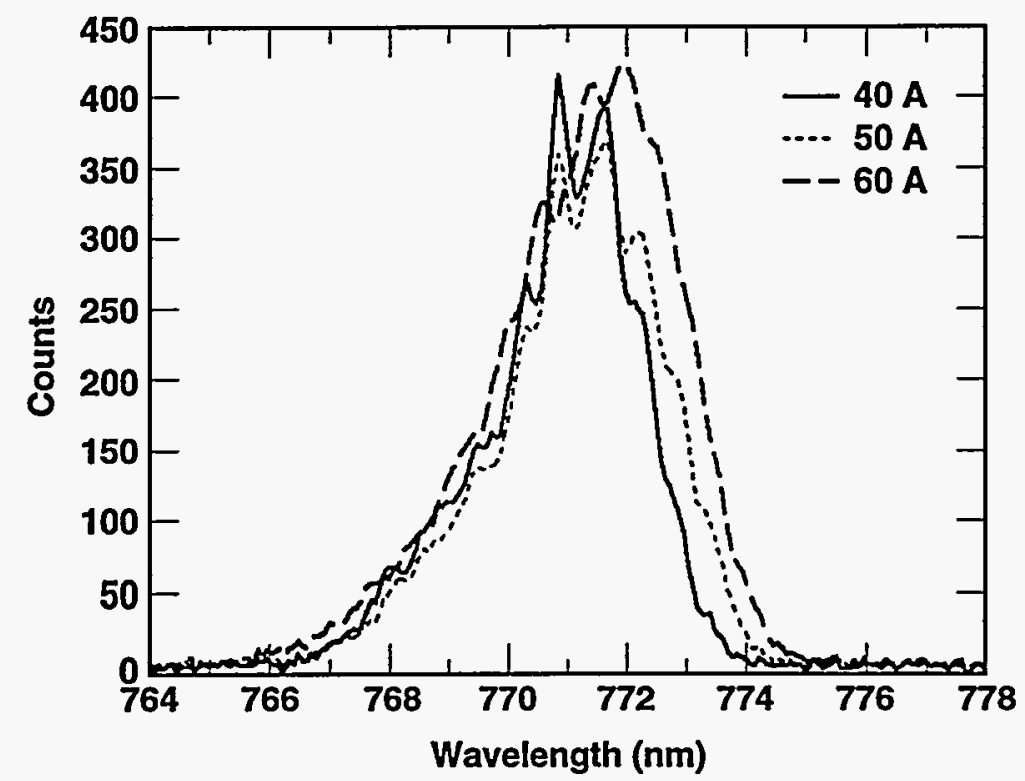

Figure 9. Plot showing comparison of laser diode spectra at different input current levels. Note the broadening of the spectrum at higher input current levels.

\section{Summary}

This compact modulator has demonstrated its ability to efficiently and accurately drive a laser diode array. The addition of the crowbar protection circuit is an invaluable addition to the integrated system and is capable of protecting the laser diode array against severe damage. We showed that the correlation between measured data and simulation indicates that our modulator model is valid and can be used as a tool in the design of future systems. The spectrometer measurements that we conducted underline the importance of current regulation to stable laser operation.

\section{Acknowledgments}

The authors would like to thank R. Beach for supplying a laser diode array. M. Norton for providing the use of an optical spectrometer. B. Moran and J. Halpin for laser performance evaluation. W. Manning for his work designing and testing various drive circuits.

This research was performed under the auspices of the U.S. Department of Energy by Lawrence Livermore National Laboratory under Contract No. W-7405-ENG-48.

\section{References}

1. Comaskey, B.J. et al . (1992), IEEE J. Quantum Electronics 28(4), 992.

2. Beach, R. et al. (1992), IEEE J. Quantum Electronics 28(4), 966 